\title{
Categorization training changes the visual representation of face identity
}

\author{
Fabian A. Soto \\ Department of Psychology \\ Florida International University \\ 11200 SW 8th St, AHC4 460, Miami, FL 33199 \\ fasoto@fiu.edu
}

\begin{abstract}
Previous research suggests that learning to categorize faces along a new dimension changes the perceptual representation of that dimension, but little is known about how the representation of specific face identities changes after such category learning. Here, we trained participants to categorize faces that varied along two morphing dimensions. One dimension was relevant to the categorization task and the other was irrelevant. We used reverse correlation to estimate the internal templates used to identify the two faces at the extremes of the relevant dimension, both before and after training, and at two different levels of the irrelevant dimension. Categorization training changed the internal templates used for face identification, even though identification and categorization tasks impose different demands on the observers. After categorization training, the internal templates became more invariant across changes in the irrelevant dimension. These results suggest that the representation of face identity can be modified by categorization experience.
\end{abstract}

Keywords: face identity; face encoding; categorization; perceptual learning

Previous research suggests that learning to categorize faces along a new dimension changes the perceptual representation of that dimension. Such studies have used stimuli varying along artificially-created morphed dimensions. A morphed face dimension is created by selecting two unfamiliar "parent" faces (Figure 1a, Step 1 ), and morphing from one parent to the other in several steps (Step 2). For example, the first level could include $0 \%$ of one face and $100 \%$ of the other, the second level $25 \%$ of one face and $75 \%$ of the other, the third level $50 \%$ of each face, and so on. To obtain a two-dimensional morph space, each of the levels in the first dimension are morphed with each of the levels in the second dimension, with each face contributing $50 \%$ to the combination (Step 3). This results in a space of faces that are varying combinations of four parent faces, as shown in Figure 1b, where each dot represents the coordinates of a face in the morph space. Such morphed dimensions are known to be integral (Soto \& Ashby, 2015); that is, different faces in the space (e.g., each point in Figure 1b) are not perceived as being composed of two dimensions, but rather as unique identities. Points in the space that are close to each other are perceived as being the same identity (e.g., the central circle of points in Figure 1b), but enough change in any direction produces a change in perceived identity (e.g., any of the outer circles of points in Figure 1b). Integrality is a desirable property, as these stimuli are used to determine what conditions lead to a change from this holistic representation to a dimensional representation. Goldstone and Steyvers (2001) found that training in a categorization task in which one morphed dimension was relevant and another was irrelevant (i.e., according to the red category bound in Figure 1b) produced such dimension differentiation, as suggested by transfer of performance to a new task in which either the relevant or the irrelevant dimension was replaced by a completely new morphed dimension. Transfer was also observed when the categorization rule was rotated by $90-$ degrees (i.e., the relevant dimension became irrelevant and vice-versa).

Other results suggest that dimension differentiation is neither a general learning phenomenon nor a stimulusspecific (i.e., similarity-based) effect, but rather a dimension-specific effect (Goldstone \& Steyvers, 2001). Performance drops more when both dimensions are replaced instead of only one (not a general learning phenomenon) and when the categorization rule is rotated 45degrees rather than 90-degrees (not a similarity-based effect, as in the former case stimulus assignment to categories is more similar to the original task). Finally, we know some of the conditions that produce this form of learning. Categorization feedback is sufficient to produce the effect, as learning occurs with circular arrangements that do not suggest any privileged direction in the morphing space (see Figure 1b). Learning of new dimensions is blocked by already-available dimensions to describe 
the categorization rule (e.g., mouth size or eyes shape). Finally, the stimulus space must be created through factorial combination of morphing sequences, as other ways of building the space produce no learning (Folstein, Gauthier, \& Palmeri, 2012).

However, little is known about the contents of this form of learning (Rescorla, 1988), and in particular how the visual representation of specific face identities changes after category learning. This is an important open issue, as the influence of categorization on face identification might help explaining the effects of social categorization (e.g., by race or sex) on face perception (Hugenberg, Young, Sacco, \& Bernstein, 2011) and more generally is informative about the influence of high-level cognition on perception (Collins \& Olson, 2014). Note that a categorization task such as that used by Goldstone and Steyvers (2001) (see Figure 1b) involves assigning stimuli that vary along identity-changing dimensions to the same category. This is similar to categorization by race or sex, albeit with much lower within-category variability. On the other hand, this is fundamentally different from learning of new identities (e.g., Jenkins, White, Van Montfort, \& Burton, 2011; Ritchie \& Burton, 2017), which involves images varying along identity-preserving dimensions such as pose, expression, illumination, age, etc. While categorization and identification might share learning mechanisms (Soto \& Wasserman, 2010), they require extracting different forms of information from faces.

How can one study changes in visual representations, which cannot be directly measured? A common strategy in psychophysics is assuming that observers use a relatively simple representation and designing experiments to estimate those representations. For example, a popular model assumes that people detect a target signal by matching an internal template containing the signal and the input stimulus (Murray, 2011). The green box in Figure 1c depicts such a simple model. In this example, the model's task is to detect a face identity. The observer matches the presented image and template using a dot product; the resulting value, which is perturbed by perceptual noise, represents the perceptual evidence that the presented image contains the target identity. The observer compares this random variable against a decision bound to make a behavioral decision (e.g., "yes" or "no").

The observer's internal template can be estimated using a reverse correlation procedure, in which participants are presented with a base image combined with random noise images (see left of Figure 1c) and asked whether they see the target identity (Observer Decision in Figure 1c). Different noise patterns are presented in each trial; the observer reports seeing the identity in some of them, but not in others. Averaging the noise images identified as the target identity produces an estimate of the template used to solve the task (see right of Figure 1c). The estimated templates, which are usually called classification images (Murray, 2011), highlight areas of the face that provide task-relevant information. Thus, different templates are useful for different tasks. For example, the templates used to determine emotional expression, gender, and identity are all different (e.g., Mangini \& Biederman, 2004; Schyns, Bonnar, \& Gosselin, 2002). That is, face categorization and identification rely on different sources of information.

Here, we used reverse correlation to estimate templates used for identification of the parents of the category-relevant dimension (A and B in Figure 1). We estimated such visual templates before and after categorization training, in the context of a task requiring participants to identify a single individual (either parent A or B) against twelve foil individuals (none of them parent faces). Templates were estimated using two different base images, represented by the two green dots in Figure 1b. These base images carried no information about the relevant dimension, but they did carry strong information about the irrelevant dimension. This allowed us to determine whether irrelevant face information influenced the templates used for identification. Four identification templates (for A and B, estimated at 2 levels of the irrelevant dimension) were estimated before and after categorization training, to evaluate the effect of such training. The templates are represented by the four arrows in Figure $1 b$, which are pointing from the base image on top of the category boundary toward the target identities. Note that these templates are defined in the space of image pixels rather than in the morphing space, and that they are estimated in the context of an identification task rather than the categorization task shown in Figure 1b. Thus, templates for two opposite identities (1-2 and 34) do not necessarily point in opposite directions, which would suggest a unified representation of the relevant dimension. Also, templates for the same identity (1-3 and 2-4) do not necessarily point in parallel directions, which would suggest a representation of the relevant dimension that is separable (i.e., invariant) from the irrelevant dimension. However, we hypothesized that categorization training would increase the degree to which both of those properties were present in the estimated internal templates.

\section{Methods}

\section{Participants}

Twenty students at Florida International University (13 females and 7 males; ages between 22 and 29 years, with median=22) participated in exchange for a monetary compensation (US\$10/hour). The study was ap- 
a.

Step 1: Select parents

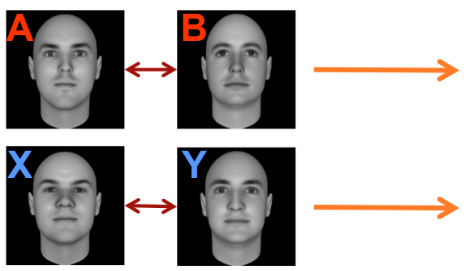

Step 3: Create two-dimensional stimuli
Step 2: Create dimensions

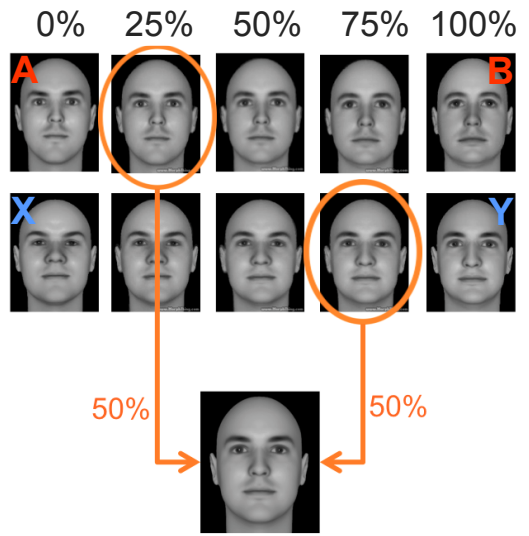

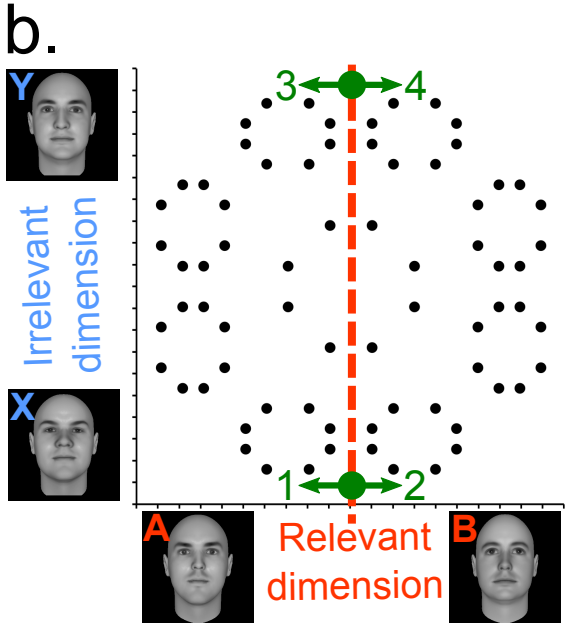
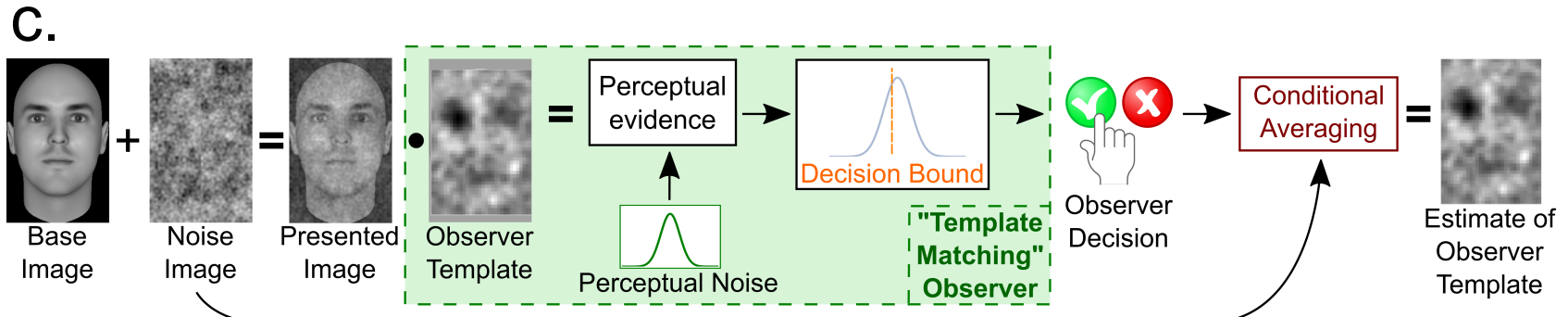

Figure 1. Tasks and stimuli used in this study. (a) Procedure used to create a two-dimensional space of morphed faces used in the categorization task. (b) Representation of the categorization task and the estimated templates in the morphing space. Points represent stimuli obtained from a specific combination of levels for each dimension. The dotted red line represents the category boundary used for training, so that stimuli on each side of the boundary were assigned to a different category. The numbered green lines represent the estimated templates, with the green dot representing the approximate position of the base image, and the arrow representing the direction of the target identity. (c) Reverse correlation technique used to estimate internal templates for face identification. The technique assumes that the observer (represented by the green box) matches incoming stimuli to an internal template. Reverse correlation allows one to estimate the template by presenting participants with noisy images and averaging those in which participants report seeing an identity. Panel (a) adapted from Cognition, Vol 139, Fabian A. Soto and F. Gregory Ashby, "Categorization training increases the perceptual separability of novel dimensions", Pages 105-129, Copyright 2015, with permission from Elsevier.

proved by an Institutional Review Board and written informed consent was obtained from all participants.

\section{Stimuli}

A detailed description of the stimuli can be found in the Supplementary Material (SM). Stimuli were created from 16 computer-generated face images. Stimuli used in the categorization task were generated using a factorial morphing procedure (Folstein et al., 2012; Goldstone \& Steyvers, 2001; Soto \& Ashby, 2015), illustrated in Figure 1a. Each dimension was obtained from a pair of parent faces (Step 1) morphed in a sequence of 19 steps (Step 2). A two-dimensional space was generated by factorially combining each of the faces in each dimension with each of the faces in the other dimension (Step 3).
These two-dimensional morphs included 50\% from each of the one-dimensional morphs. The specific stimuli presented to participants in the categorization task are represented by the black points in Figure 1b. The base images presented during the reverse correlation task are represented by the green points in Figure 1b. An additional 12 faces were included during identification training in the reverse correlation task. All images in the reverse correlation task were combined with noise masks, created using a weighted sum of sinusoids as proposed by Mangini and Biederman (2004).

\section{Procedure}

Participants completed four sessions of a reverse correlation task and three sessions of categorization training. 
A detailed description of the tasks is included in the $S M$.

The experiment started with two 90-minute sessions of the reverse correlation task. A session involved identification of one parent of the relevant dimension (A or B; see Figure 1b); each parent was identified in a different session. Participants were instructed to identify which of two images was more similar to the parent face. They were warned that the two faces would be very similar in most trials. Participants completed ten blocks, each comprising 36 identification training trials followed by 100 reverse correlation trials, for a total of 360 identification training trials and 1,000 reverse correlation trials. Due to a coding error early in the experiment, two participants completed 6 rather than 10 blocks of the task.

Two different faces were presented during each identification training trial, each combined with a different noise pattern. One image contained the target face (parent A or B) and the other included one of twelve foils. The participant's task was to identify which of the two images contained the target, by pressing the "left arrow" or "right arrow" keys. A choice was followed by "CORRECT" in blue font color or "INCORRECT" in red font color. Each distractor face was repeated three times within a block. The order of trials and the left-right position of each face within a trial were random.

During a reverse correlation trial, participants were presented with a choice between the same base image combined with a pattern of noise and its reversed pattern (i.e., noise $\times-1$ ). The base images (green points in Figure 1b) contained ambiguous information about the category-relevant dimension $(50 \%$ from each parent face). Different base images were used in different blocks, and they contained strong information about the category-irrelevant dimension (i.e., $100 \%$ from one parent face and $0 \%$ from the other).

After completion of the two initial reverse correlation sessions, participants completed three one-hour sessions of the categorization task depicted in Figure 1b, where the black dots represent the morphed stimuli presented and the red dotted line represents their separation into two different categories. A single face was presented in each trial, and participants reported whether it belonged to category A or B through a key press, which was always followed by feedback about the correctness of the response. Each session consisted of 9 blocks of 72 trials each, for a total of 648 trials. Each stimulus (36 per category) was presented once in a block, with the order randomized within the block.

After categorization training, all participants completed two new reverse correlation sessions, identical to those completed before categorization training.

\section{Data Analysis}

See $S M$ for details about inclusion/exclusion criteria, data analysis software, and template estimation and analysis.

The main analysis was performed on data from the reverse correlation task. A rejection criterion of $\alpha=.05$ was used.

Individual templates and anti-templates were estimated using the $\mathrm{R}$ package rcicr (Dotsch \& Todorov, 2012; Mangini \& Biederman, 2004). We estimated four templates (for faces A and B at 2 levels of the irrelevant dimension) per participant and their corresponding anti-templates, both before and after training. Templates are estimated by averaging noise related to selected (template) or not selected (anti-template) noise patterns. The final templates are grayscale images with a single intensity value at each pixel.

Two similarity measures were obtained from each participant's set of templates and anti-templates, using the Pearson correlation of corresponding pixel intensity values in the two images, within an oval-shaped face area mask that excluded non-facial features. The first similarity measure was computed between templates for two different identities (the two parent faces of the relevant dimension, A and B) estimated using the same base image (i.e., same level of the irrelevant dimension). Using the numeration from Figure $1 \mathrm{~b}$, template 1 was correlated with anti-template 2 , and template template 3 was correlated with anti-template 4. Higher similarity here would indicate a stronger dimensional representation, with the templates for identities $\mathrm{A}$ and $\mathrm{B}$ pointing in opposite directions. The second similarity measure was computed between templates for the same identity (i.e., same parent face of the relevant dimension), but estimated using different base images (i.e., different values in the irrelevant dimension). Using the numeration from Figure 1b, template 1 was correlated with template 3 , and template 2 was correlated with template 4 . Higher similarity here would indicate representations of the identities that were more invariant to (or separable from) changes in the irrelevant dimension.

Both similarity measures were analyzed with a 2 (learning stage: before vs. after categorization training) $\mathrm{x}$ 2 (dimension level) within-subjects ANOVA. Partial etasquared $\left(\eta_{p}^{2}\right)$, along with a $90 \%$ confidence interval on this estimate, was calculated as a measure of effect size.

Average templates were computed for each combination of level of the relevant dimension, level of the irrelevant dimension, and pre- versus post-categorizationtraining conditions. Separability images were obtained by subtracting two average templates for identification of the same face (A or B), estimated at different levels of the irrelevant dimension: template 1 minus 3 , and tem- 
a.

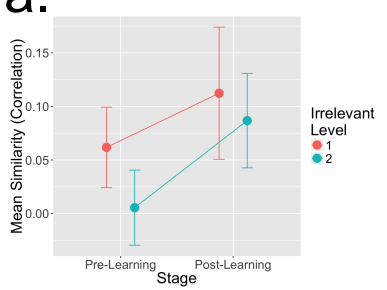

C. Relevant Level 1

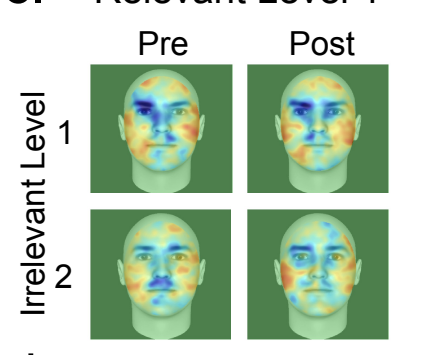

d.

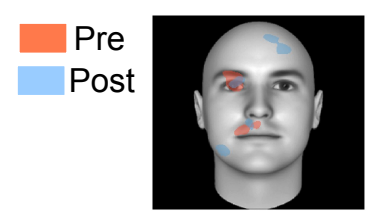

b.

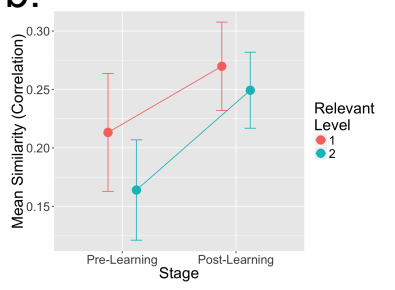

Relevant Level 2
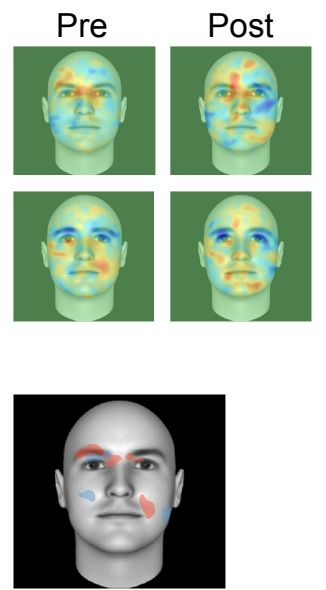

Figure 2. Experimental results. (a) Mean similarity between the internal template of a face at one extreme of the relevant dimension and the anti-template of the face at the other extreme of the relevant dimension; higher values represent a stronger dimensional representation. (b) Mean similarity between the internal template for the same face (one extreme of the relevant dimension) at different levels of the irrelevant dimension; higher values represent an increase in invariance across changes in the irrelevant dimension. (c) Average templates estimated in this study; different colors represent whether more (red) or less (blue) luminance would produce identification of the target face (green is neutral). (d) Facial areas represented differently across changes in the irrelevant dimension, both before (Pre, in red) and after (Post, in blue) categorization training. Error bars represent standard errors of the mean.

plate 2 minus 4 . The two separability images were obtained both pre- and post-categorization-training. Separability images were tested for significance using a twotailed cluster test from Random Field Theory (Chauvin, Worsley, Schyns, Arguin, \& Gosselin, 2005).

\section{Results}

After obtaining classification images, we obtained two measures summarizing changes in these templates that could be produced by categorization training. First, the

templates of the two parents of the relevant dimension (A and B) could become negatively correlated, as it has been observed with natural face categories (Dotsch \& Todorov, 2012), indicating a stronger dimensional representation in which such extremes are encoded as opposite values. Categorization training had an effect in this direction (Figure 2a), but it was not significant, $F(1,15)$ $=3.03, p>.1, \eta_{p}^{2}=.168[.000, .410]$. This result could be due to low statistical power, but our data does not allow us to reach a conclusion regarding this point (see power analysis in $S M)$. The effect of irrelevant level, $F(1,15)$ $=1.32, p>.1, \eta_{p}^{2}=.080[.000, .317]$ and the interaction, $F(1,15)=.5, p>.1, \eta_{p}^{2}=.032[.000, .244]$, were also not significant. Second, the templates for the same identity at different levels of the irrelevant dimension could become more similar (i.e., correlated), which would explain why categorization training increases invariance of the relevant dimension (i.e., perceptual separability; see Soto \& Ashby, 2015). Our results show a significant effect of training in this direction (Figure $2 b$ ),$F(1,15)=5.95$, $p=.028, \eta_{p}^{2}=.284[.019, .509]$. There was no effect of relevant level, $F(1,15)=1.044, p>.1, \eta_{p}^{2}=.065[.000$, $.296]$, or the interaction, $F(1,15)=.098, p>.1, \eta_{p}^{2}=.006$ $[.000, .161]$. An exploratory analysis (for details, see $S M$ ) revealed that identification training was unlikely to have contributed to these results.

In sum, the main result of our study is that categorization training influences the internal templates used for face identification, which become more invariant to changes in face information that is irrelevant for categorization. Importantly, previous research has shown that identification and categorization tasks impose different demands on an observer (Schyns et al., 2002). In an ideal observer analysis, we corroborated that the two tasks used here demanded sampling from different areas of the image to be solved optimally (for details, see $S M$ ).

To explore more precisely what changes in internal templates produced such effect, we computed average templates (Figure 2c) and subtracted templates for identification of the same face (A or B), estimated at different levels of the irrelevant dimension: template 1 minus 3 , and template 2 minus 4 (see Figure 1b). Next, we obtained significant clusters from these difference images (Chauvin et al., 2005), representing face areas used differently across changes in the irrelevant dimension. Figure $2 \mathrm{~d}$ displays significant clusters before (red) and after (blue) categorization training. Comparing these clusters allows us to determine what changes in the internal templates underlie the increase in invariance observed earlier.

Note first that some clusters that are significant before categorization training disappear or show a reduction in size after training. Clusters that do not disappear show a reduction in their extension (volume reduced by $62.52 \%$ 
in eye cluster and $60.11 \%$ in mouth cluster of Figure 2dleft, and by $59.42 \%$ in eye cluster of Figure 2d-right), but not in their location $(91.47 \%, 30.14 \%$ and $39.75 \%$ overlap of the post-training cluster with the corresponding pre-training cluster). The fact that some significant clusters are similarly located before and after categorization training suggest that such training did not radically change sampling of facial information. This is confirmed by looking at the classification images in Figure $2 \mathrm{c}$, and by a positive correlation between corresponding templates (e.g., template 1) before and after categorization training (mean correlations for templates 1-4: .19, $.17, .18$, and .18; all $p<.001$ uncorrected).

The observed reduction in number and size of significant clusters helps explaining the main result shown in Figure $2 b$. Importantly, these clusters are all on or near internal face features (eyes and mouth), which are areas that are known to provide information for identification (Butler, Blais, Gosselin, Bub, \& Fiset, 2010; Schyns et al., 2002). On the other hand, some significant clusters appear only after categorization training, and they tend to be located around external face features (forehead, jaw line, and cheeks), which people tend to ignore in face identification (Butler et al., 2010; Schyns et al., 2002). This suggests that perhaps the main effect of categorization training was to increase template separability (i.e., similarity of templates across changes in the irrelevant dimension) around the internal face features, but to decrease such separability around external face features. An exploratory analysis revealed that not to be the case, as a similar increase in similarity was observed in internal and external face areas (for details, see $S M$ ).

In conclusion, our study suggests that categorization training changes the internal templates used for face identification. The most important change involved an increase in the invariance of these templates to changes in the irrelevant dimension (Figure 2b), which explains previously-observed increments in perceptual separability (Soto \& Ashby, 2015). While such increase in invariance was observed across the face, the effect seemed to be particularly strong around internal face features, where significant violations of separability either disappeared or decreased in extent.

\section{Discussion}

Categorization training changed the internal templates used for face identification, even though identification and categorization tasks impose different demands on an observer (Schyns et al., 2002). After categorization training, the internal templates used to identify a face became more invariant across changes in the irrelevant dimension, which relates to previously-observed increments in dimensional separability (Soto \& Ashby, 2015). Such increase in invariance was observed across the face, but the effect seemed particularly strong around internal face features, which are known to provide important information for identification (Butler et al., 2010; Schyns et al., 2002).

However, categorization training did not dramatically change the face information sampled for identification. Many features of the internal templates remained relatively unchanged after categorization training (Figure $2 \mathrm{c}$ ), and some areas of the face represented differently across changes in the irrelevant dimension were similarly located before and after training (Figure 2d). One interesting possibility is that the identity representations already-existing before categorization training were used as a "scaffold" for the development of new representations after categorization training.

Our results suggest that the representation of individual face identities can be modified by categorization experience, opening the possibility that naturalistic categorization tasks (e.g., by race, sex, age, etc.) have a similar effect. The influence of categorization experience on internal face templates might thus explain the multiple effects of social categorization on face perception (Hugenberg et al., 2011) or why internal templates of emotional expression vary with cultural experience (Jack, Caldara, \& Schyns, 2012). An important caveat is that naturalistic tasks involve much larger variation in identity than what we can obtain using the morphing procedure common to studies of dimensional differentiation. More research is needed to understand to what extent the two paradigms are comparable.

Our results might also inform studies about learning of new faces across changes in identity-preserving variables (e.g., Jenkins et al., 2011; Ritchie \& Burton, 2017). Although the present study involved images varying along identity-changing morphed dimensions, in both cases the task is technically one of categorization, in which multiple images are assigned the same label (i.e., either an individual or a group of individuals) and the participant must learn to extract the label-predicting information while ignoring the label-irrelevant information in faces. There is reason to believe that a common learning mechanism might underlie such learning across category levels (Soto \& Wasserman, 2010). An important question for future research is whether the representation of face identities learned from multiple identitypreserving images (rather than from a single image, as in the present study) is affected by categorization training. Thus, the current study is only a first step toward understanding the influence of categorization on identity representation. Future research will require richer stimulus sets and estimated representations (e.g., by assuming more complex observer models).

In sum, dimensional differentiation through categorization may have important consequences for face per- 
ception in naturalistic circumstances. The present study contributes to our understanding of the contents of this form of perceptual learning (Rescorla, 1988), by showing how it changes the visual representation of individual faces.

\section{Acknowledgements}

S.D.G. This work was supported in part by an NIMH Grant to F.A.S. (1R21MH112013-01A1). The author thanks Jefferson Salan and Claudia Wong for their help collecting data for this study.

\section{Open Practices Statement}

The data and materials for all experiments are available at https://osf.io/dy23v/ and none of the experiments was preregistered.

\section{References}

Butler, S., Blais, C., Gosselin, F., Bub, D., \& Fiset, D. (2010). Recognizing famous people. Attention, Perception, $\mathcal{E}$ Psychophysics, 72(6), 1444-1449. doi: 10.3758/APP.72.6.1444

Chauvin, A., Worsley, K. J., Schyns, P. G., Arguin, M., \& Gosselin, F. (2005). Accurate statistical tests for smooth classification images. Journal of Vision, 5(9), 1.

Collins, J. A., \& Olson, I. R. (2014, August). Knowledge is power: How conceptual knowledge transforms visual cognition. Psychonomic Bulletin and Review, 21(4), 843-860. doi: 10.3758/s13423-013 -0564-3

Dotsch, R., \& Todorov, A. (2012). Reverse correlating social face perception. Social Psychology and Personality Science, 3(5), 562-571.

Folstein, J. R., Gauthier, I., \& Palmeri, T. J. (2012). Not all morph spaces stretch alike: How category learning affects object discrimination. Journal of Experimental Psychology: Learning, Memory and Cognition, 38(4), 807-802.

Goldstone, R. L., \& Steyvers, M. (2001). The sensitization and differentiation of dimensions during category learning. Journal of Experimental Psychology: General, 130(1), 116.

Hugenberg, K., Young, S. G., Sacco, D. F., \& Bernstein, M. J. (2011). Social categorization influences face perception and face memory. In A. J. Calder, G. Rhodes, M. H. Johnson, \& J. V. Haxby (Eds.), The Oxford Handbook of Face Perception (pp. 245-261). Oxford University Press.

Jack, R. E., Caldara, R., \& Schyns, P. G. (2012). Internal representations reveal cultural diversity in expectations of facial expressions of emotion. Journal of Experimental Psychology: General, 141(1), 1925.

Jenkins, R., White, D., Van Montfort, X., \& Burton, A. M. (2011). Variability in photos of the same face. Cognition, 121(3), 313-323.

Mangini, M. C., \& Biederman, I. (2004). Making the ineffable explicit: estimating the information employed for face classifications. Cognitive Science, 28(2), 209-226.

Murray, R. F. (2011, May). Classification images: A review. Journal of Vision, 11(5), 2. doi: 10.1167/ 11.5.2

Rescorla, R. A. (1988, March). Pavlovian conditioning: It's not what you think it is. American Psychologist, 43(3), 151-160.

Ritchie, K. L., \& Burton, A. M. (2017). Learning faces from variability. The Quarterly Journal of Experimental Psychology, 70(5), 897-905.

Schyns, P. G., Bonnar, L., \& Gosselin, F. (2002). Show me the features! Understanding recognition from the use of visual information. Psychological Science, 13(5), 402-409.

Soto, F. A., \& Ashby, F. G. (2015, June). Categorization training increases the perceptual separability of novel dimensions. Cognition, 139, 105-129. doi: 10.1016/Journalcognition.2015.02.006

Soto, F. A., \& Wasserman, E. A. (2010). Missing the forest for the trees: Object discrimination learning blocks categorization learning. Psychological Science, 21(10), 1510-1517. doi: 10.1177/ 0956797610382125 


\title{
Supplementary Material for "Categorization training changes the visual representation of face identity"
}

\author{
Fabian A. Soto
}

\section{Contents}

Supplementary Methods 1

Stimuli . . . . . . . . . . . . . . . . . . . . . . . . . . . . 1

Parent and Foil Faces . . . . . . . . . . . . . . . . . . . . . . . . 1

Morphed Face Stimuli . . . . . . . . . . . . . . . . . . . . . . . . . . . . 2

Noise Patterns for Reverse Correlation . . . . . . . . . . . . . . . . . . . . . . . . . . 2

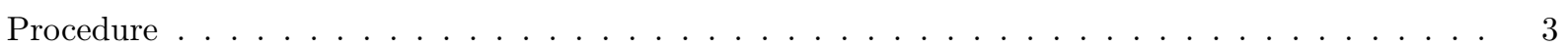

Identification Task and Reverse Correlation . . . . . . . . . . . . . . . . . . . . . . 3

Categorization Task . . . . . . . . . . . . . . . . . . . . . . . . . 5

Data Analysis . . . . . . . . . . . . . . . . . . . . . . . . . . 5

Software . . . . . . . . . . . . . . . . . . . . . . . . 5

Exclusion/Inclusion Criteria . . . . . . . . . . . . . . . . . . . . . . . 5

Template Estimation and Analysis . . . . . . . . . . . . . . . . . . . . . 5

Face Masks . . . . . . . . . . . . . . . . . . . . . . . . . . . . . . . 6

Supplementary Results 6

Ideal Observer Analysis . . . . . . . . . . . . . . . . . . . . . . . . . . . . . . 6

Analysis of Similarity Measures Including Only Internal or External Face Features . . . . . . . . . 8

Analysis of the Effect of Identification Training on Similarity Measures . . . . . . . . . . . . . . . . 9

References

\section{Supplementary Methods}

\section{Stimuli}

\section{Parent and Foil Faces}

The stimuli were created from images chosen from a database of 300 computer-generated Caucasian faces described by Oosterhof and Todorov (2008), created using the Facegen Modeller program (http://facegen.com), Version 3.1. From the original database, 30 male faces were chosen that had similar eyebrow color and similar levels of facial fat.

Similarity ratings were obtained for these stimuli in a pilot study, using an efficient method described by Goldstone (1994). Briefly, twelve participants were presented with all 30 male faces in a single screen, ordered in a 5 (columns) $\times 6$ (rows) grid located at the bottom-right of the screen. The 30 faces were randomly located in this grid at the beginning of the experiment. Participants were given the following instructions:

You will be shown a set of faces on the screen. You should move the faces depending on how similar they are to other faces. The more similar two faces are, the closer they should be. To choose where to place each face, take into account how similar it is to all other faces and move it to the place were it is close to faces that are very similar to it and far from faces that are very dissimilar to it. 
After reading these instructions, participants were allowed to move the 30 faces anywhere in the monitor. They were given unlimited time, and were finished only after they were satisfied with the position of all the faces. From each participant's final configuration, we recorded the coordinates of each face in units of pixels, and used those coordinates to compute the euclidean distance between all pairs of faces. The distances were then normalized by dividing each of them by the largest distance recorded from the participant. Thus, final distances ranged from zero to one for all participants. The faces that would be used as parents to our morphed dimensions (see section below) were chosen based on these final dissimilarity values.

Dimensions are created by morphing from one face to another, meaning that each dimension had two parent faces. These parent faces were chosen so that their dissimilarity was relatively equivalent across the two dimensions; this way, the two dimensions would not differ widely in salience. The chosen parents for the first dimension (category-relevant) had a mean dissimilarity of 0.51 (S.D. $=0.13$ ) and the chosen parents for the second dimension (category-irrelevant) had a mean dissimilarity of 0.49 (S.D. $=0.16$ ). These values were well within $15 \%$ of each other, which was the pre-set criterion that we chose before the study. A second criterion was that the parent pairs should not be discriminable along an easily verbalizable dimension, such as degree of femininity/masculinity or head width. These steps were taken to ensure that the final stimuli shown to the participants did not have a clear dimensional structure; rather, the dimensional structure of these stimuli had to be learned through categorization training.

From the remaining faces not chosen as parents for morphing, 12 were randomly selected to serve as foils in the identification task. These 12 faces are shown in the right of Figure S2a, under the title "Foils".

All images were converted to grayscale and their intensity histograms were equalized, to ensure that stimuli along the resulting morphing dimensions varied in shape features, but not in simpler features such as skin color and brightness.

\section{Morphed Face Stimuli}

We used a factorial morphing procedure (Goldstone and Steyvers, 2001; Folstein et al., 2012; Soto and Ashby, $2015)$ to generate morphed faces that are combinations of the four parent faces. The procedure is illustrated in Figure 1a of the main manuscript. After choosing two pairs of parent faces (Step 1; see section Parent and Foil Faces), each dimension is created by generating morphs with different proportions from each pair of parents (Step 2). Here, dimensions were created using a continuous sequence of 19 morphs for each pair of parents, with percentages of parent 2 equal to $0 \%, 6 \%, 14 \%, 20 \%, 24 \%, 30 \%, 32 \%, 38 \%, 42 \%, 50 \%$, $58 \%, 62 \%, 68 \%, 70 \%, 76 \%, 80 \%, 86 \%, 94 \%$, and $100 \%$. Finally, a two-dimensional space was generated by factorially combining each of the faces in each dimension with each of the faces in the other dimension (Step 3). These two-dimensional morphs included $50 \%$ from each of the one-dimensional morphs.

Figure $1 \mathrm{~b}$ from the main manuscript displays the coordinates in the two-dimensional space of the stimuli presented to participants. The coordinates of the morphed faces presented during the categorization task are represented by black dots in the figure. Their circular configuration was used to de-emphasize the dimensional structure of the stimuli (Goldstone and Steyvers, 2001; Folstein et al., 2012; Soto and Ashby, 2015) and make sure that new directions in the morphed space were learned via feedback during the categorization task. The coordinates of the base images used during the reverse correlation task are represented by green dots in the same figure.

\section{Noise Patterns for Reverse Correlation}

Noise patterns were created and added to base images using the rcicr $\mathrm{R}$ package $\mathrm{v}$. 0.3.2. This software creates noise patterns through a weighted sum of sinusoidal patterns (as first proposed by Mangini and Biederman, 2004), where the weights are random variables that change in each trial.

The procedure used to create a single noise image is schematized in Figure S1. The noise image results from the combination of several basis images shown in the leftmost column of Figure S1. These are sinusoidal patterns with six different orientations, and two different phases (only one phase is shown for each orientation, the second phase is omitted). Each one of the basis images was multiplied by a random weight, and the resulting weighted images were added together to produce a "noise tile." The red box in Figure S1 represents the process of generating a single noise tile. Several of these tiles are generated and scaled as shown in the red arrows of Figure S1. Tiles that are scaled down are added together to produce a full-scale image, which 
results in five patterns of noise at different scales. These five patterns are added together to produce the final noise image, which was presented overlaying a base face during a reverse correlation trial.

Unique noise patterns were created as shown in Figure S1 for each trial and each participant.

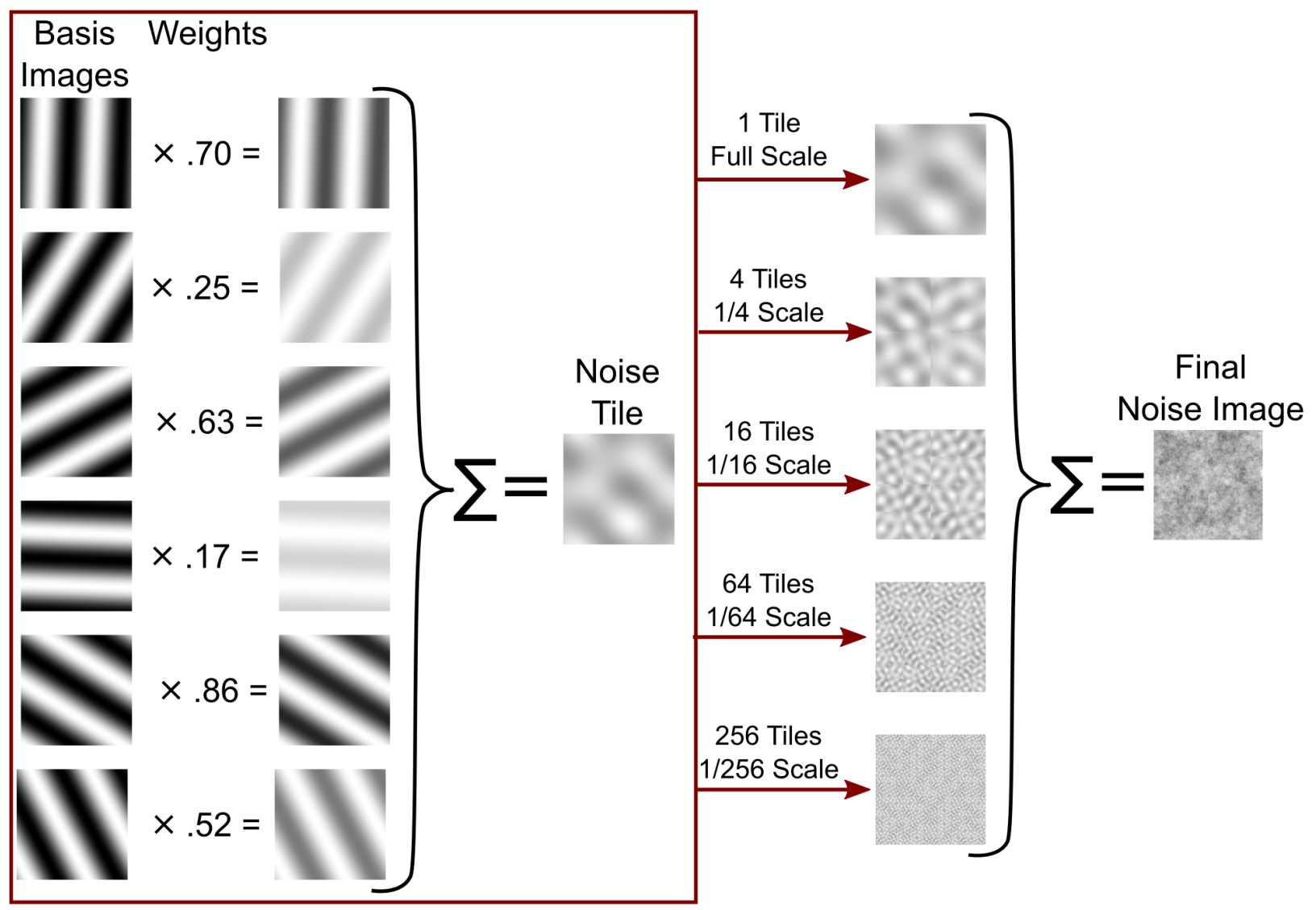

Figure S1: Procedure used to generate noise patterns. Figure by F. Soto, shared under creative commons license at https://figshare.com/articles/Noise_sampling_procedure/7603331

\section{Procedure}

All tasks were programmed in Psychopy2 Experiment Builder version 1.82.01 (Peirce, 2007, 2009).

\section{Identification Task and Reverse Correlation}

Participants completed two 90-minute sessions of a reverse correlation task before categorization training, and identical sessions after categorization training. Each of the two sessions involved identification of a different target face, which could be one of the two parents of the category-relevant dimension. These two faces are shown in the left column of Figure S2a, under the title "Target". One of the sessions involved identification of parent $\mathrm{A}$ against several other faces, without any presentations of parent $\mathrm{B}$, and the other session involved identification of parent B against several other faces, without any presentations of parent A. The two sessions were run within the span of two weeks, and separated by at least one hour. Most sessions (about $80 \%$ ) were separated by $1-4$ days.

In each session, participants first received instructions indicating that their task was to identify which of two images was more similar to a target face, and were allowed to study the target face shown without any noise for as long as they wanted. They were warned that the two faces would be very similar in most trials, and that in such cases they should do their best to choose the face most similar to the target face. 


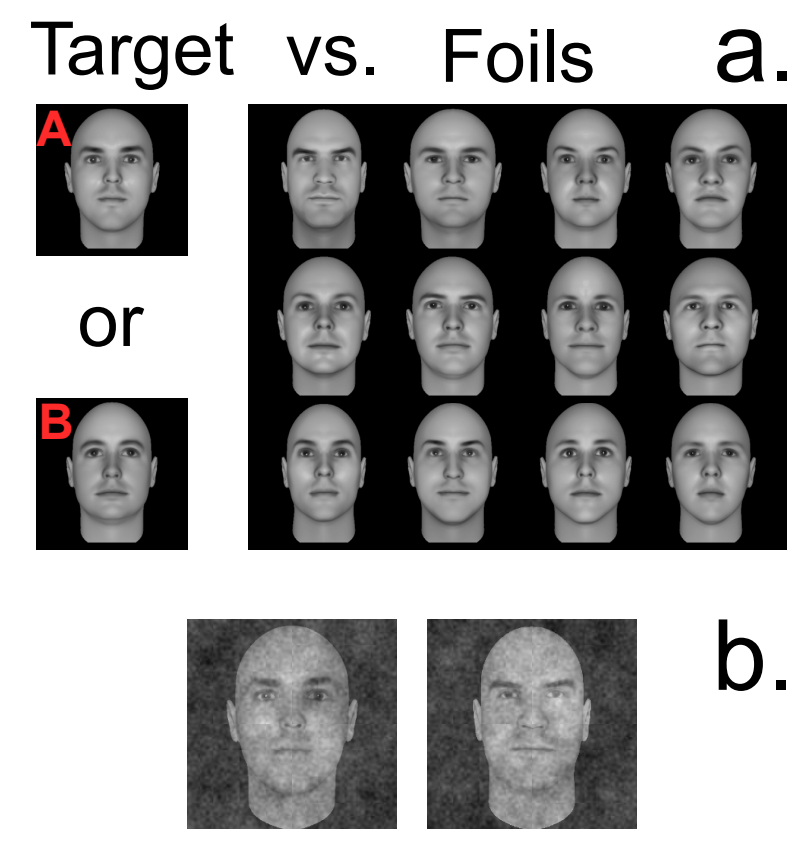

Figure S2: (a) Targets and foils in the identification task and (b) an example of the stimulus pairs presented during a single trial from training in the identification task. The participant's task was to indicate which of the two noisy stimuli was the target face, and ignore the foil face.

After instructions, participant completed ten blocks, each comprising 36 identification training trials followed by 100 reverse correlation testing trials, for a total of 360 identification training trials and 1,000 testing trials in total. Two participants completed 6 rather than 10 blocks of the task.

In each identification training trial, participants were presented with two images of faces embedded in noise, side-by-side as shown in Figure S2b. One of the faces was the target face (parent A or B, see left of Figure S2a) and the other was one of twelve foil faces (i.e., one of the faces in the right of Figure S2a). The participant's task was to identify which of the two faces was more similar to the target face, by pressing the "left arrow" or "right arrow" keys. The participant's choice was followed by feedback consisting of the word "CORRECT" in blue font color or the word "INCORRECT" in red font color. Each distractor face was repeated three times within a block. The order of trials and the left-right position of each face within a trial were randomly determined.

In each reverse correlation testing trial, participants were presented with two versions of the same base face image. The two images differed only in the pattern of noise superimposed to them. One of them included a random pattern of noise, constructed as described in section Noise Patterns for Reverse Correlation, and the other included the opposite pattern of noise.

There were two possible base images, obtained from the morphed face space used during the categorization task, and their coordinates are shown as green dots in Figure 1b of the main manuscript. As shown in that figure, the base images were averaged (i.e., level 50\%) along the relevant dimension, landing exactly in the boundary between the two categories and thus carrying no information about them. On the other hand, the two base images were positioned in the two extremes of the irrelevant dimension (i.e., either $0 \%$ or $100 \%$ level in the irrelevant dimension), and thus they carried a strong signal difference along that dimension. The two base images were used in different blocks and led to two different estimates of the internal template used for identification of the target image; this allowed to determine how information about the irrelevant dimension might influence such template. 


\section{Categorization Task}

Participants were exposed to three one-hour sessions of training in the categorization task shown in Figure $1 \mathrm{~b}$ of the main manuscript. The sessions were run within a span of three days and no more than two sessions were run on the same day. Consecutive sessions were separated by at least 1 hour and at most 25 hours. Each session consisted of 9 blocks of 72 trials each, for a total of 648 trials.

At the beginning of each session, instructions were displayed on the screen indicating that the participant's task was to categorize faces as accurately as possible into two different categories (clubs) based purely on physical appearance. The instructions also explained the structure of each trial and how to report a categorization response. Participants were warned that they would need to guess the correct answer early in training, but they would get more accurate as the experiment progressed. Sessions were divided in blocks of 72 trials each. Each stimulus (36 per category) was presented once in a block, with the order randomized within the block.

Each trial started with the presentation of a white cross in the middle of a black screen for $500 \mathrm{~ms}$. Immediately afterwards a face stimulus was presented in the middle of the black screen until the participant pressed one of the two response buttons in the keyboard or a time deadline of $2 \mathrm{~s}$ was reached, whichever happened first. Participants could press the keys D or K in their keyboard, which were re-labeled "A" and "B", respectively. After a key press, the participant received feedback about the correct response. For correct responses, the word CORRECT was presented for $500 \mathrm{~ms}$, in green font color in the middle of the screen. For incorrect responses or if the time deadline was reached, the word INCORRECT was presented for 500 $\mathrm{ms}$, in red font color in the middle of the screen. This was followed by a $1 \mathrm{~s}$ inter-trial interval, during which the monitor was completely black.

\section{Data Analysis}

\section{Software}

Analyses were performed using R v. 3.2.1 (R Core Team, 2015) extended with the packages rcicr v. 0.3.2 (https://cran.r-project.org/web/packages/rcicr/), spatialfil v. 0.15 (https://cran.r-project.org/web/ packages/spatialfil/index.html), and ggplot2 v. 2.1.0 (http://ggplot2.org/), running in RStudio v. 0.99.486 (https://www.rstudio.com/). Cluster tests from Random Field Theory were performed using the stat4CI toolbox (Chauvin et al., 2005) running in Matlab R2013b (8.2.0.701; Copyright The Mathworks, Inc.).

\section{Exclusion/Inclusion Criteria}

The main analysis was performed on data from the reverse correlation task. Pre-training data was included only if the participant was above $85 \%$ correct during identification training trials. Post-training data was included if participants met both the previous criterion and showed a performance level above $65 \%$ correct in the last block of categorization training. This is a low criterion that allowed to exclude only participants that did not show any signs of learning. All these criteria were set before performing the main analyses.

All participants passed the criterion for inclusion of pre-training data, most with accuracies above $90 \%$ correct $($ mean $=97.02 \%, \mathrm{sd}=3.74 \%)$ in identification training. The post-training data of three participants were excluded. Participants included in the analysis showed mean accuracy of $81.17 \%(\mathrm{sd}=7.58 \%)$ in the last block of the categorization task, and mean accuracy of $97.59 \%(\mathrm{sd}=2.19 \%)$ in identification training.

\section{Template Estimation and Analysis}

Individual templates and anti-templates were estimated using the rcicr $\mathrm{R}$ package v. 0.3.2. This software creates noise masks through a weighted sum of sinusoidal patterns (as first proposed by Mangini and Biederman, 2004), where the weights are random variables that change in each trial (for a detailed description, see section Noise Patterns for Reverse Correlation). Templates are estimated first in the space of weights, by averaging weights related to selected (template) or not selected (anti-template) noise patterns. The averaged weights are then used to transform the template to image space through the same process used to generate noise patterns. 
Due to a recording error, data for level one of the relevant dimension was missing from a single participant during pre-training. Each template was computed using 500 trials, except for the templates of two participants who contributed only 300 trials due to a coding error early in the experiment that was swiftly corrected. With the procedure used here, about 300 trials produce reliable template estimates (Dotsch and Todorov, 2012; Mangini and Biederman, 2004).

Average templates were computed for each combination of level of the relevant dimension, level of the irrelevant dimension, and pre- versus post-categorization-training conditions. Each individual image was first smoothed using a Gaussian filter with a standard deviation of four pixels. Smoothing is a common procedure among studies using classification image techniques to study face perception (Dotsch and Todorov, 2012; Éthier-Majcher et al., 2013; Wang et al., 2011). The assumption behind this procedure is that most signals of interest in the templates extend across several contiguous pixels, which is in line with studies showing that people rely mostly on intermediate spatial frequency information for face identification (with most studies reporting between 8 and 20 cycles per face width; see Gold et al. 1999; Gao and Maurer 2011). Our choice of a standard deviation of four pixels should help increasing the signal-to-noise ratio around this relevant scale, although it is difficult to know in advance exactly what is the optimal choice for the width of this filter (Chauvin et al., 2005).

The smoothed templates were transformed to $z$-scores by subtracting the mean and dividing by the standard deviation from the area outside the face mask, as recommended by Chauvin et al. (2005). Finally, the average template was obtained by summing individual templates and dividing the result by the square root of the number of participants (Éthier-Majcher et al., 2013).

Separability images were obtained by subtracting two average templates for identification of the same face (A or B), estimated at different levels of the irrelevant dimension: template 1 minus 3, and template 2 minus 4 . The two separability images were obtained both pre- and post-categorization-training.

Separability images were tested for significance using a two-tailed cluster test from Random Field Theory (Chauvin et al., 2005). The justification for the choice of this test is similar as that given above for spatial filtering of estimated templates. The test is sensitive to signals that occupy many contiguous pixels, which is just the kind of signal that we would expect in face identification.

\section{Face Masks}

Face masks used in the analysis of similarity measures were manually created using the image manipulation software Gimp v. 2 (https://www.gimp.org). They were saved as JPEG images and loaded in R as matrices of ones and zeros. Figure S3 shows the area of the face covered by each mask.

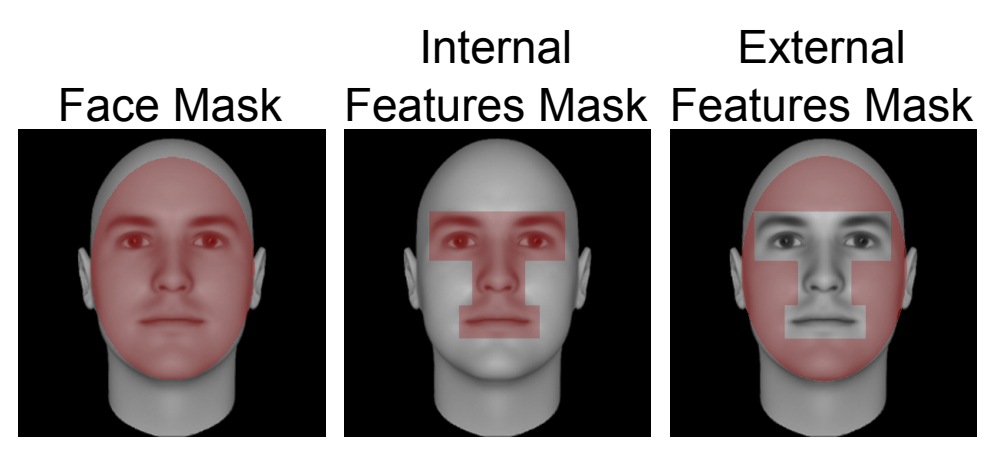

Figure S3: Area covered by the face masks (in red) in different data analyses.

\section{Supplementary Results}

\section{Ideal Observer Analysis}

An important aspect of our findings is that face categorization training changed the internal templates used in a face identification task. This result is interesting because the demands of both tasks are different, and previous research suggests that human observers use different templates to solve each of them (Mangini 
and Biederman, 2004; Schyns et al., 2002). That is, as the demands of the identification task are different from those of the categorization task, there is no reason to expect that categorization training would change the templates used for identification, as this change would lead to a potentially sub-optimal strategy in the identification task.

One possibility is that the specific categorization and identification tasks used here produce similar demands on the observer. In that case, observers might be able to detect the similar demands of both tasks and use a single template across tasks. That is, after learning of the categorization task and the corresponding template, participants could modify their identification templates as well, as they would know that discriminating face A (or B) from foil faces in Figure S2 requires similar information as categorizing faces on the basis of how much of face A (or B) they contain.

To determine whether the specific categorization and identification tasks used here produce similar demands on observers, we performed an ideal observer analysis of those tasks.

The ideal observer model used in our simulations was developed by Tjan et al. (1995) and it is often used as a benchmark in visual categorization studies. The model allows one to determine which aspects of observed behavior can be explained as resulting directly from task and stimulus demands and not from any visual processing carried out by participants. The model assumes an observer that knows all possible training stimuli and their assigned labels (i.e., responses). Upon presentation of an image $R$, the task of the observer is to choose label $L_{i}$ that maximizes $p\left(L_{i} \mid R\right)$. Each label $L_{i}$ is assigned to several images, which are kept in the observer's memory and are represented by $M_{i j}$, where $j$ indexes different images. If the memory images $M_{i j}$ and the currently observed image $R$ are represented as vectors of luminance values (i.e., pixels), and under the assumption that image $R$ is generated from a single object, perturbed by the addition of Gaussian noise with zero mean and standard deviation $\sigma$, then Tjan et al. (1995) have shown that maximizing $p\left(L_{i} \mid R\right)$ is equivalent to maximizing the following:

$$
L^{\prime}(i)=\sum_{j} \exp \left(-\frac{1}{2 \sigma^{2}}\left\|R-M_{i j}\right\|^{2}\right) p\left(M_{i j}\right) .
$$

That is, the optimal strategy is to compute the sum of a similarity measure between $R$ and each memory $M_{i j}$, weighted by the probability of observing image $M_{i j}$ in a given trial of the task.

A different ideal observer was used for the two identification tasks (identification of A against all foil faces, and identification of B against all foil faces; see Figure S2) and for the categorization task. The first identification task had parent A labeled $L_{1}$ and all the foil faces labeled $L_{2}$. The second identification task had parent B labeled $L_{1}$ and all the foil faces labeled $L_{2}$. The categorization task had stimuli closer to parent A labeled $L_{1}$ and stimuli closer to parent B labeled $L_{2}$.

Each ideal observer was presented with 10,000 trials with images from each label, for a total of 20,000 trials. Independent Gaussian noise with zero mean was added to each pixel of the images shown to the ideal observer. The value of the noise standard deviation, $\sigma$, was determined individually for each task, to bring performance of the observer down to $75 \%$ correct. The noise statistics were available to the ideal observer, which compared the resulting image against all of the training images included in the task, giving as output the category label with the highest value of $L^{\prime}(i)$ from Equation 1. The obtained noise images and responses were used to estimate the ideal observer's internal template using the following equation (Murray, 2011):

$$
\mathbf{t}=\left(\overline{\mathbf{n}}_{12}+\overline{\mathbf{n}}_{22}\right)-\left(\overline{\mathbf{n}}_{11}+\overline{\mathbf{n}}_{21}\right),
$$

where $\overline{\mathbf{n}}_{i j}, i=1,2$ and $j=1,2$, is the average of noise patterns presented together with an image with label $C_{i}$, to which the ideal observer responded with label $C_{j}$.

The estimated internal templates were smoothed using a Gaussian filter with a standard deviation of four pixels. The smoothed templates were transformed to $z$-scores by subtracting the mean and dividing by the standard deviation. The resulting templates are shown in Figure S4, plotted on top of the average face from the categorization task. It can be seen that the areas weighted more heavily by the ideal observer are different in different tasks. For example, identification of face A heavily weighted information around the right eye (strongest red). The same feature is not observed in the template for categorization, which in turn weights heavily information around the left eyebrow that is not important for identification.

To quantify the similarity of the different templates, we also report their correlations in the arrows of Figure S4. All correlations were negative and very close to zero, suggesting that indeed all three tasks 


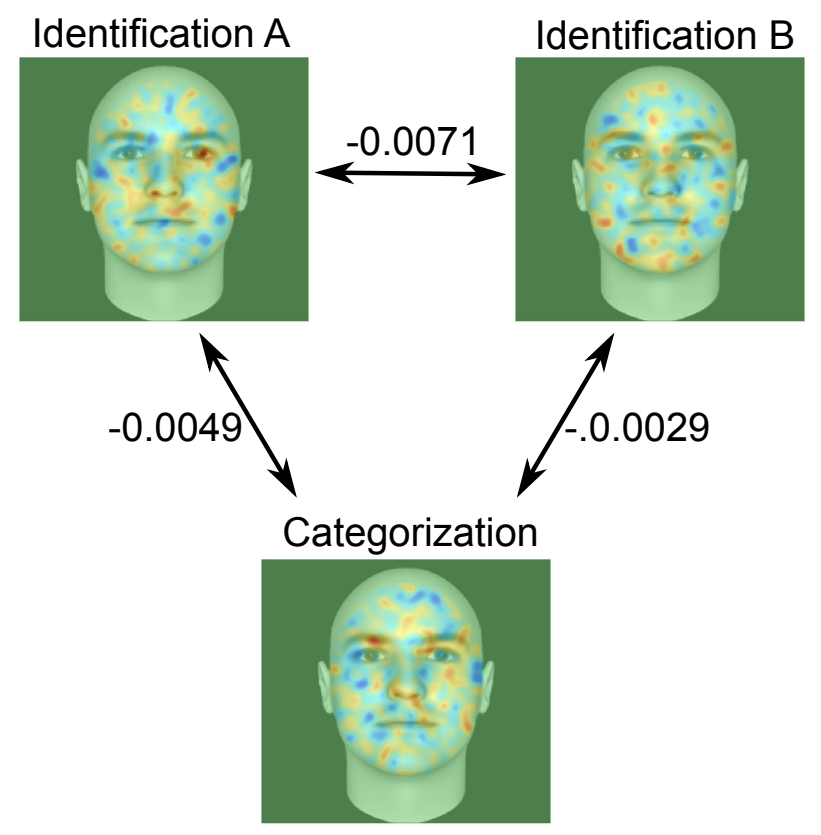

Figure S4: Ideal observer templates for the identification and categorization tasks used in this study.

require using information from different areas of the face differently. None of the correlations in Figure S4 were significant according to a two-sided $t$-test (all $p>0.1$ ).

\section{Power Analysis of the Effect of Categorization Training on the Dimensionality Test}

Based on previous studies on the effect of categorization training on dimension differentiation (Folstein et al., 2012; Goldstone and Steyvers, 2001) and separability (Soto and Ashby, 2015), we expected that the current study would find a similar effect on the measures of dimensionality and separability. While we observed an increase in both measures after categorization training, that increase was statistically significant only for the test of separability, but not for the test of dimensionality. A possibility that should be considered is that our study was underpowered to detect the effect of categorization training on dimensionality. Here, we performed a post-hoc power analysis for that effect using G*Power v. 3.1.9.3 (http://www.gpower.hhu.de/). Given that the observed effect size was $\eta_{p}^{2}=0.168$ with a $90 \%$ confidence interval ranging from 0.000 to 0.410 , the power of this test was only 0.39 when estimated using the effect size point-estimate, but could range anywhere from 0.00 to 0.92 when one considers the lower and upper limits of the confidence interval. That is, if one assumes that the point-estimate of the effect size is accurate, then the study had a statistical power well below the common standard of 0.8 power. On the other hand, if one takes into account the uncertainty around the point-estimate of effect size, then the study had a statistical power anywhere from close to zero to well above the 0.8 standard.

Assuming that the point-estimate of the effect size is accurate, one would need a sample size of 41 to achieve the desired power of 0.8 , but this value can be anywhere from 14 to several thousand when one takes into account the full range of effect sizes within the $90 \%$ confidence interval.

\section{Analysis of Similarity Measures Including Only Internal or External Face Fea- tures}

The analysis of separability images reported in the main manuscript revealed that significant deviations of separability existed both before and after categorization training (see red and blue clusters in Figure 2d of the main manuscript). With categorization training, significant clusters around the internal face features 
(i.e., the area around the eyes and mouth) disappeared or reduced their size, whereas new clusters appeared in outer areas of the face (i.e., jaw line, cheeks, and forehead). This suggests that perhaps the main effect of categorization training is to increase template separability (i.e., similarity of the template for a given identity across changes in the irrelevant dimension) only around the internal face features (i.e., the area around eyes, nose and mouth), but to decrease template separability in external face features. We performed an exploratory analysis to determine whether there was any evidence for such a dissociation between face areas, by creating two new masks that selected pixels around internal and external features of the face (see Figure S3), and computing separate similarity measures using each mask.

The results are shown in Figure S5. They are shown in the same format as Figure $2 \mathrm{a}$ and $2 \mathrm{~b}$ from the main manuscript, to facilitate comparison. The most important result is that the pattern of results observed when only internal or external face features are included in the analyses of similarities was in general very similar to that observed in the main analysis. In all cases, the effect of categorization training is to increase similarity measures. To determine whether there was any effect of the mask used (internal vs. external) on the results, both similarity measures were analyzed with a 2 (learning stage: before vs. after categorization training) x 2 (dimension level) x 2 (mask: internal vs. external) within-subjects ANOVA. This withinsubjects ANOVA was identical as that used in the main analysis, except for the addition of mask as a new factor.

We discuss the analysis of separability first, as this was the main motivation of the current exploratory analysis (Figure S5b and S5d). The analysis revealed a main effect of mask, $F(1,15)=33.11, p<.001$, reflecting the fact that the mean similarities were higher for internal features (panel b) than for external features (panel d). There was also a significant main effect of categorization training, $F(1,15)=6.46$, $p<.05$, mirroring the same effect from the main analysis. No other effects were significant in this analysis. Importantly, the interaction between categorization training and mask was not significant, $F(1,15)=1.79$, $p>$.1. In sum, there is no evidence that the effect of categorization training was different for external and internal features; an increase in similarity is observed in both cases, despite the fact that significant clusters appear after categorization training in external areas of the face (see Figure $2 \mathrm{~d}$ in the main manuscript).

No effects were significant in the analysis of dimensionality (Figure S5a and S5c). As in the main analysis, categorization training produced an increase in similarity measures, but this effect did not reach significance, $F(1,15)=3.96, p>.05$. More importantly, there was no main effect or interaction involving mask.

\section{Analysis of the Effect of Identification Training on Similarity Measures}

Our study focuses on the question of whether categorization training changes the internal templates used for face identification. For this, we used a pre-post design in which participants served as their own controls. One of the issues with a pre-post design is that it is not clear whether a difference in some measured behavior before and after training is due to the training itself versus the passage of time, a general improvement in performance due to experience with the experimental setting, familiarity with faces, etc.

As indicated in the main manuscript, previous research has shown that the effect of categorization on dimension differentiation is not the result of such factors (Goldstone and Steyvers, 2001; Folstein et al., 2012). Our study is based on that previous research, and builds upon it by focusing on unanswered questions related to the contents of this form of learning.

Still, our experiment was different from the previous literature in that participants received training in an identification task both before and after the categorization training sessions. Because of this, participants had more identification training in their second reverse correlation session, after categorization training, than in their first reverse correlation session, before categorization training. Thus, an important question is whether identification training could have influenced the templates used for face identification. There are three reasons to believe this not to be the case.

First, there is no reason to expect that identification training would have an effect on either template separability or dimensionality. Both tests were developed to further explore previously-observed effects of categorization training, which are not observed after simple identification training. For example, categorization training increases discriminability along the category-relevant dimension, but not along other dimensions in the morphing space (Folstein et al., 2012; Goldstone and Steyvers, 2001). This dimensional differentiation led us to hypothesize that the internal representations of the two parent faces would be more negatively correlated after categorization training, which was measured through the dimensionality test. 

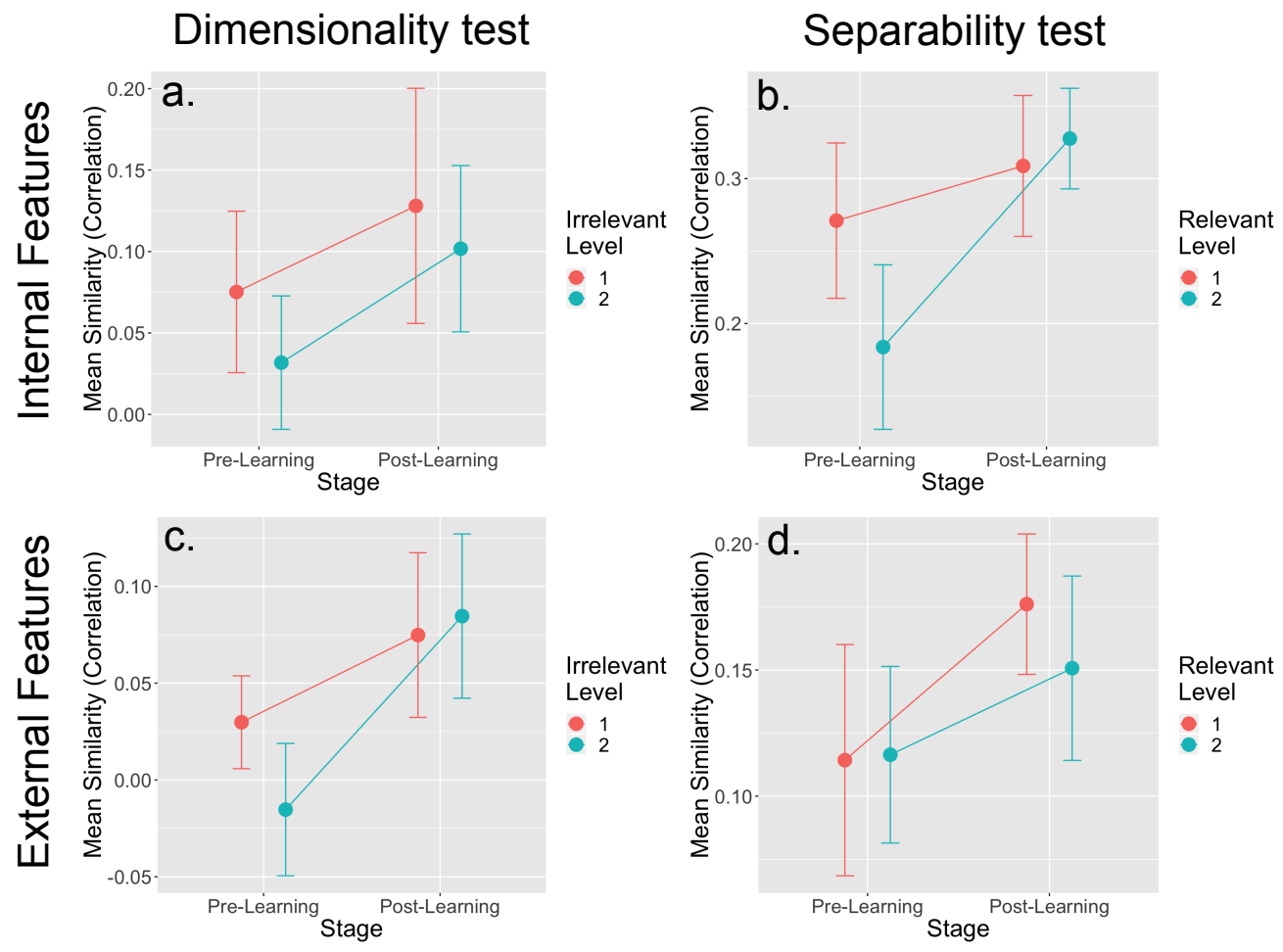

Figure S5: Results of the analysis of similarity measures restricted to areas of the face containing internal (panels a and b) or external features (panels c and d).

Similarly, categorization training increases the separability of the category-relevant dimension from variations in an irrelevant dimension (Soto and Ashby, 2015). This increase in separability or invariance led us to hypothesize that the internal representation of a parent face would become more invariant to changes in the category-irrelevant dimension after categorization training, which was measured through the separability test.

Second, performance in the identification tasks was at ceiling during the pre-categorization session. The mean accuracy was $97.02 \%(\mathrm{sd}=3.74 \%)$ before categorization training and $97.59 \%(\mathrm{sd}=2.19 \%)$ after categorization training; the small difference of $0.57 \%$ was not significant, $t(16)=-0.2853, p>0.1$. The same result was observed when the two identification tasks were analyzed separately. This suggests that any effect of identification training would be present both before and after categorization training.

Third, an exploratory analysis found no effect of identification training on the dimensionality and separability tests. We re-analyzed our data by estimating internal templates using the first and last 150 reversecorrelation trials in a session. We repeated this analysis for the data before and after categorization training. If identification training had an effect in internal templates, then some evidence of that effect should be observable when comparing these early and late trials of identification training.

The results are shown in Figure S6. They are shown in a format similar to that of Figures 2a and 2b from the main manuscript, but here the main effect under consideration (displayed in the $x$-axis) is that of identification training. The data has been separated into internal templates computed before categorization training (Figure S6a and b) and internal templates computed after categorization training (Figure S6c and d). 

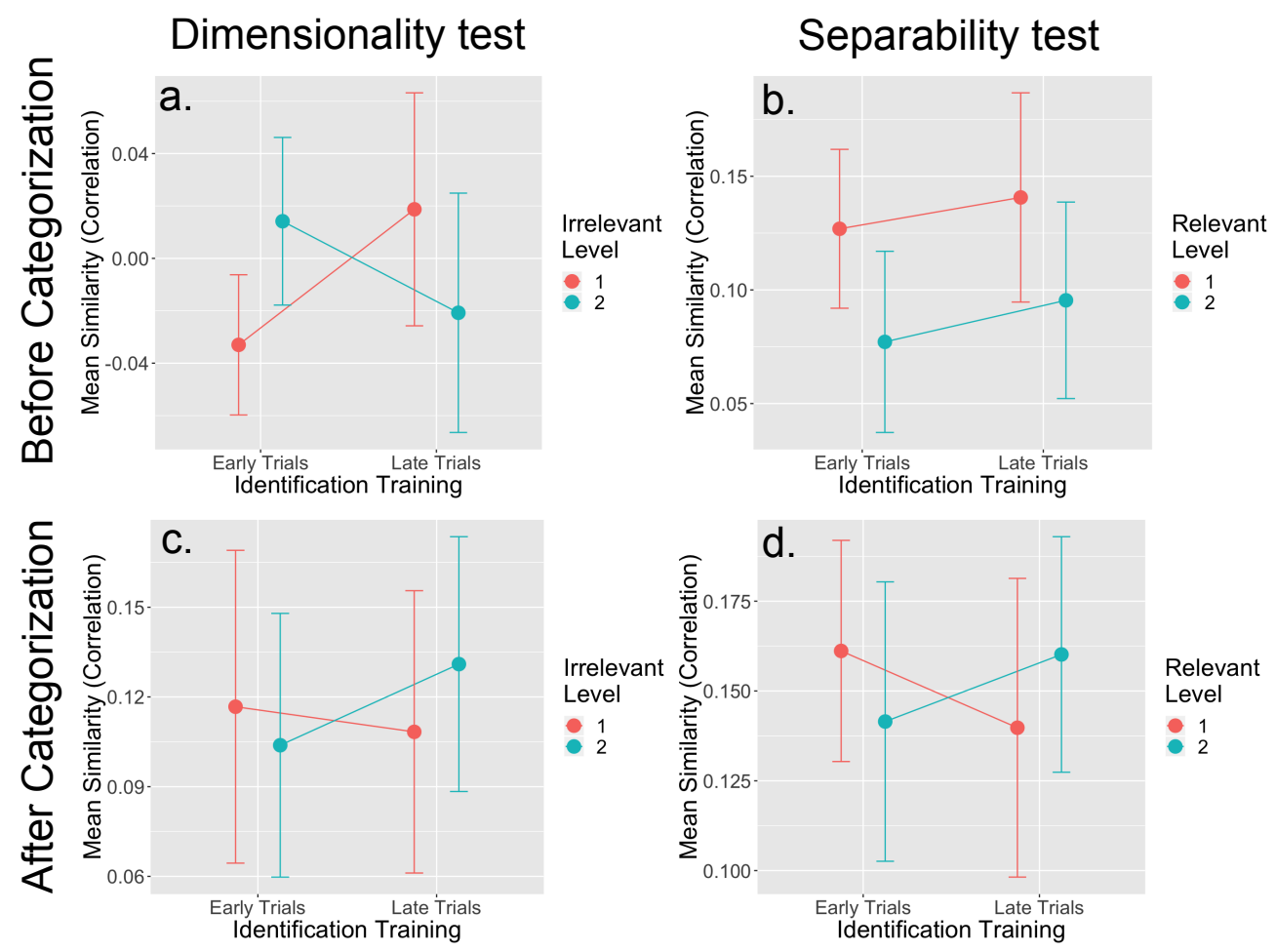

Figure S6: Results of the analysis of similarity measures when influence of identification training is reduced.

We attempted several analyses to determine whether a reliable effect of identification training could be found in our data. We first entered the data from each of the panels in Figure S6 to an ANOVA with identification training (early trials vs. late trials) as one of the main factors, and either irrelevant level (in the analysis of dimensionality) or relevant level (in the analysis of separability) as the other main factor. The main effect of identification training was not significant in any of these analyses (all $p>.1$ ). This can be easily observed in Figure S6, where the effect of identification training is either inconsistent across levels of the second factor (increasing similarity at one level, but decreasing at the other; see panels a, c, and d) or there was a very small increase in similarity with identification training (see panel b; the effect is on average 1-2\%, accompanied with standard errors about 4 times larger). In addition, the interaction between identification training and either relevant or irrelevant level was also not significant in any of the analyses (all $p>.1$ ).

In addition, to determine whether we could find an effect of identification training when pooling the results before categorization and after categorization, we pooled the data in the columns of Figure S6 (a and $\mathrm{c}$ for the Dimensionality test; $\mathrm{b}$ and d for the Separability test). The data were entered into an ANOVA similar to the previous ones. Again, identification training had no significant main effect or interaction (all $p>.1$ ).

Finally, to determine whether using a different number of trials for the computation of templates might reveal some evidence of an influence of identification training, the previous analyses were repeated using 100 , 200 and 250 trials. We did not observe a main effect of identification training or an interaction involving identification training in any of these exploratory analyses.

Overall, our data did not provide any evidence of an effect of identification training on template separability or dimensionality, as assessed through similarity measures.

\section{References}

Chauvin, A., Worsley, K. J., Schyns, P. G., Arguin, M., and Gosselin, F. (2005). Accurate statistical tests for smooth classification images. Journal of Vision, 5(9):1. 
Dotsch, R. and Todorov, A. (2012). Reverse correlating social face perception. Social Psychological and Personality Science, 3(5):562-571.

Éthier-Majcher, C., Joubert, S., and Gosselin, F. (2013). Reverse correlating trustworthy faces in young and older adults. Frontiers in Psychology, 4:592.

Folstein, J. R., Gauthier, I., and Palmeri, T. J. (2012). Not all morph spaces stretch alike: How category learning affects object discrimination. Journal of Experimental Psychology: Learning, Memory, and Cognition, 38(4):807-802.

Gao, X. and Maurer, D. (2011). A comparison of spatial frequency tuning for the recognition of facial identity and facial expressions in adults and children. Vision Research, 51(5):508-519.

Gold, J., Bennett, P. J., and Sekuler, A. B. (1999). Identification of band-pass filtered letters and faces by human and ideal observers. Vision Research, 39(21):3537-3560.

Goldstone, R. L. (1994). An efficient method for obtaining similarity data. Behavior Research Methods, $26(4): 381-386$.

Goldstone, R. L. and Steyvers, M. (2001). The sensitization and differentiation of dimensions during category learning. Journal of Experimental Psychology: General, 130(1):116.

Mangini, M. C. and Biederman, I. (2004). Making the ineffable explicit: estimating the information employed for face classifications. Cognitive Science, 28(2):209-226.

Murray, R. F. (2011). Classification images: A review. Journal of Vision, 11(5):2.

Oosterhof, N. N. and Todorov, A. (2008). The functional basis of face evaluation. Proceedings of the National Academy of Sciences, 105(32):11087-11092.

Peirce, J. W. (2007). PsychoPy-Psychophysics software in Python. Journal of Neuroscience Methods, $162(1-2): 8-13$.

Peirce, J. W. (2009). Generating stimuli for neuroscience using PsychoPy. Frontiers in Neuroinformatics, $2: 10$.

R Core Team (2015). R: A language and environment for statistical computing.

Schyns, P. G., Bonnar, L., and Gosselin, F. (2002). Show me the features! Understanding recognition from the use of visual information. Psychological Science, 13(5):402-409.

Soto, F. A. and Ashby, F. G. (2015). Categorization training increases the perceptual separability of novel dimensions. Cognition, 139:105-129.

Tjan, B. S., Braje, W. L., Legge, G. E., and Kersten, D. (1995). Human efficiency for recognizing 3-D objects in luminance noise. Vision Research, 35(21):3053-3069.

Wang, H. F., Friel, N., Gosselin, F., and Schyns, F. G. (2011). Efficient bubbles for visual categorization tasks. Vision Research, 51(12):1318-1323. 